Open Access to Pharmaceutical and Medical Research

C 2011-18, publisher and licensee JDDT, This is an Open Access article which permits unrestricted non-commercial use, provided the original work is properly cited

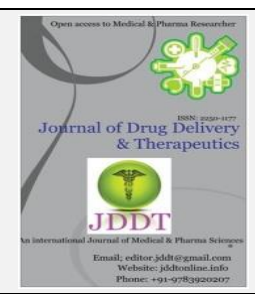

Open Access

Research Article

\title{
Formulation and Evaluation of Bilayer Matrix Tablets of Nebivolol Hydrochloride and Valsartan
}

\author{
Selvi Arunkumar 1*, Dr. L. Srinivas ${ }^{2}$, Dr. D. Satyavati ${ }^{3}$ and Dr. C. Emmanuel ${ }^{4}$ \\ 1. Assistant professor, Gleneagles Global Health city, Perumbakkam, Chennai, Tamil Nadu, India. \\ 2 Professor, GITAM Institute of Pharmacy, GITAM deemed to be University, Visakhapatnam, A.P. India. \\ 3. Principal, Department of Pharmacy, Brilliant Group of Technical Institutions, Abdullapur, Hyathnagar, Hyderabad, Telangana, India. \\ 4. Director, Academics and Research, Gleneagles Global Health City, Chennai, Tamil Nadu, India.
}

\begin{abstract}
The present study is an attempt to develop bilayer matrix tablets of Nebivolol Hydrochloride and Valsartan with immediate release for Nebivolol Hydrochloride and sustained release for Valsartan. Superdisintegrants such as sodium starch glycolate and Crosscarmellose sodium were evaluated for immediate release of Nebivolol Hydrochloride and polymers HPMC K100M and K4M for sustained release of Valsartan. Preformulation studies were performed prior to compression. The compressed bilayer tablets were evaluated for weight variation, thickness, hardness, friability, drug content and in vitro drug release using USP dissolution apparatus type 2 in $0.01 \mathrm{~N} \mathrm{HCl}$ and phosphate buffer pH 6.8 . All the pre and post compression parameters were found to be within the acceptable limits. The results of dissolution show that the formulations B3 was the best of all immediate and sustained release layer batches. The release kinetics of Valsartan was subject to curve fitting analysis in order to identify the best fit kinetic model. The regression analysis proves that the best formulations follow zero order release and drug release by diffusion process based on Fick's law of diffusion. The data for stability studies infer no considerable change in drug co ntent and dissolution rates as per ICH guidelines. The best formulation B3 was subjected to in vivo pharmacokinetic studies in rabbit model. In vitro, In vivo correlation (IVIVC) showed considerable linearity. Hence a novel bilayer tablet formulation of Nebivolol Hydrochloride and Valsartan was successfully developed by combining both immediate (IR) and sustained (SR) release layers.
\end{abstract}

Keywords: Bilayer tablets, fixed unit dosage form, Nebivolol hydrochloride, Valsartan, LC-MS analysis.

Article Info: Received 06 June 2019; $\quad$ Review Completed 18 July 2019; $\quad$ Accepted 24 July 2019; Available online 15 August 2019

Cite this article as:

Arunkumar S, Srinivas L, Satyavati D, Emmanuel C, Formulation and Evaluation of Bilayer Matrix Tablets of Nebivolol

Hydrochloride and Valsartan, Journal of Drug Delivery and Therapeutics. 2019; 9(4-s):82-93

http://dx.doi.org/10.22270/jddt.v9i4-s.3257

*Address for Correspondence:

Selvi Arunkumar, Assistant professor, Gleneagles Global Health city, Perumbakkam, Chennai, Tamil Nadu, India.

\section{INTRODUCTION}

Developing a new molecule is expensive and time consuming. Other alternative .other alternative methods such as individualizing drug therapy, dose titrations, therapeutic drug monitoring and novel drug delivery system, using old drug by improving safety and efficacy ratio, brings out promising results. Successes in drug delivery research are the result of concerned efforts by the scientist of variety of disciplines who recognize the need and potentiate for improving pharmcotherapeutics through the development of novel drug delivery systems.

The drug delivery systems are usually known by terms like sustained, controlled, targeted, smart, intelligent, novel, therapeutics and programmed. However the basic rationale for these varied delivery modules is the alteration or manipulation of the pharmacokinetic and pharmacodynamics of pharmacologically active moieties. This can be achieved through novel drug delivery devices. ${ }^{1}$

Essential or primary hypertension is a persistent elevation of blood pressure which is not caused by underlying cardiac, endocrine or renal diseases. 2 Approximately $70 \%$ of blood pressure elevations in youth represent early onset of essential hypertension.2,3 Attaining a target blood pressure can be very difficult with monotherapy especially in case of patients with multiple drug therapy. A survey on trends in the use of antihypertensive agents in France from 2002 to 2012 supported the view of suboptimal treatment for hypertension with monotherapy and hence prescriptions of fixed dose combinations from $19 \%$ to $30 \%{ }^{3}$ 
Fixed dose combination refers to the combination of two or more active ingredients in a single pharmaceutical formulation. ${ }^{4}$ A number of clinical trials show that most hypertensive patients were unlikely to achieve a normal pressure by taking single drug for a quite a long time. ${ }^{5}$

Formulation technologies such as multilayer tablets, multiparticulate systems, active film coating and hot melt granulation are developed in the design of formulation. Reviews of physicians' desk reference show that infectious, cardiovascular, hormones, allergies and pain are the top most five medical areas for fixed dose combinations of oral dosage forms, nevertheless hypertension treatment takes $80 \%$ in cardiovascular. 6

\section{Rationale for drug selection}

Valsartan is an angiotensin II receptor antagonist that is used in the treatment of hypertension. It act by blocking the binding of angiotensin II and I to its receptors thereby blocking vasoconstrictor and aldosterone secreting effect of angiotensin II selectively. The most preferred route for this drug is oral delivery in the form of tablets. Valsartan has poor water solubility, low bioavailability (approximately 20$25 \%$ ) and short half-life (nearly hrs.) which makes it an ideal candidate for sustained release. Hence in the present work this ideology is adopted.7

Nebivolol is a novel beta1-blocker with a greater degree of selectivity for beta1- adrenergic receptors than other agents in this class and a nitric oxide (NO)-potentiating, vasodilatory effect that is unique among beta-blockers currently available to clinicians .A NO-potentiating agent such as Nebivolol may have an important role in hypertensive populations with reduced endothelial function such as diabetics, Nebivolol is unique among beta blockers in that, at doses $<10 \mathrm{mg}$, it does not inhibit the increase in heart rate normally seen with exercise. The efficacy of Nebivolol has been tested successfully in clinical trials against other agents including other beta-blockers, angiotensin-converting enzyme-inhibitors and calcium channel antagonists in patients with hypertension, angina, and congestive heart failure. The tolerability of Nebivolol has been shown to be superior to that of atenolol and Metoprolol. ${ }^{8}$
Multilayer tablets give a successful development of controlled release formulation and provide a way of successful drug delivery system. Conventional dosage form sometimes leads to undesirable toxicity and poor efficiency due to fluctuation in concentration in blood tissues.

For the current research Nebivolol Hydrochloride in immediate release and Valsartan in sustained release layer was selected as fixed dose combination for bilayer tablet technology. Recently, fixed dose combination of Nebivolol hydrochloride, a selective $\beta 1$ antagonist and valsartan an angiotensin II receptor blocker was approved by USFDA for hypertension. Pharmacological profiles of Nebivolol hydrochloride and valsartan alone and in combination are well characterized. In addition, a large 8-week randomized trial in stages I-II hypertensive patients $(\mathrm{N}=4161)$ demonstrated greater blood pressure-reducing efficacy for $33 \mathrm{Neb} /$ valsartan SPCs than component monotherapies with comparable tolerability. In a biomarkers sub study $(\mathrm{N}=805)$, Nebivolol/valsartan single-pill combination prevented valsartan-induced increases in plasma renin, and a greater reduction in plasma aldosterone was observed with the highest single-pill combination dose vs. valsartan 320 mg/day. ${ }^{9}$

\section{MATERIALS AND METHODS}

Nebivolol hydrochloride and Valsartan were received as gift samples from Apotex Pharmachem India Pvt. Ltd. The polymers such as Hydroxypropyl Methylcellulose K100M(Methocel K100M premium), K4M(Methocel K4M premium), Dibasic calcium Phosphate(anhydrous, FCC), Sodium Starch Glycolate(731713H),Croscarmellose sodium(Ac di sol NF), FD\&C blue lake, microcrystalline cellulose, silicon dioxide(Aerosil 200) and magnesium stearate(ligamed MF) were obtained from KMS Pharma Formulation development Healthcare Pvt Ltd.

\section{Formulation of Bi-layer tablets:}

The ingredients after sifting, Prelubrication and blending was subjected for precompression parameters like excipient compatibility studies (Table 1, 2), Angle of Repose, Bulk Density, Tapped Density Carr's. Index (\%), Hausner's Ratio. ${ }^{10}$ the results are shown in table 3and 4. The FTIR studies were done for standard Nebivolol HCl, Valsartan and Excipients.

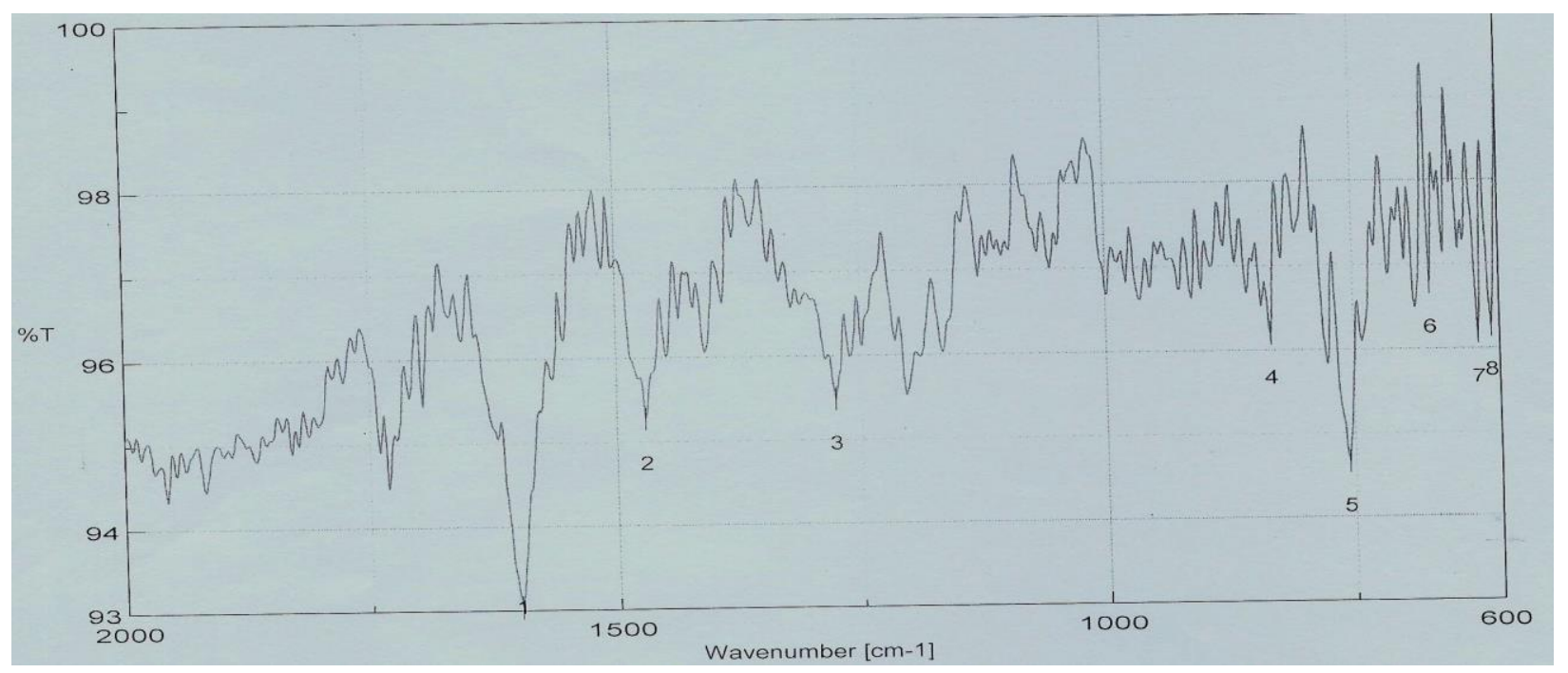

Figure 1: IR spectrum of Valsartan 


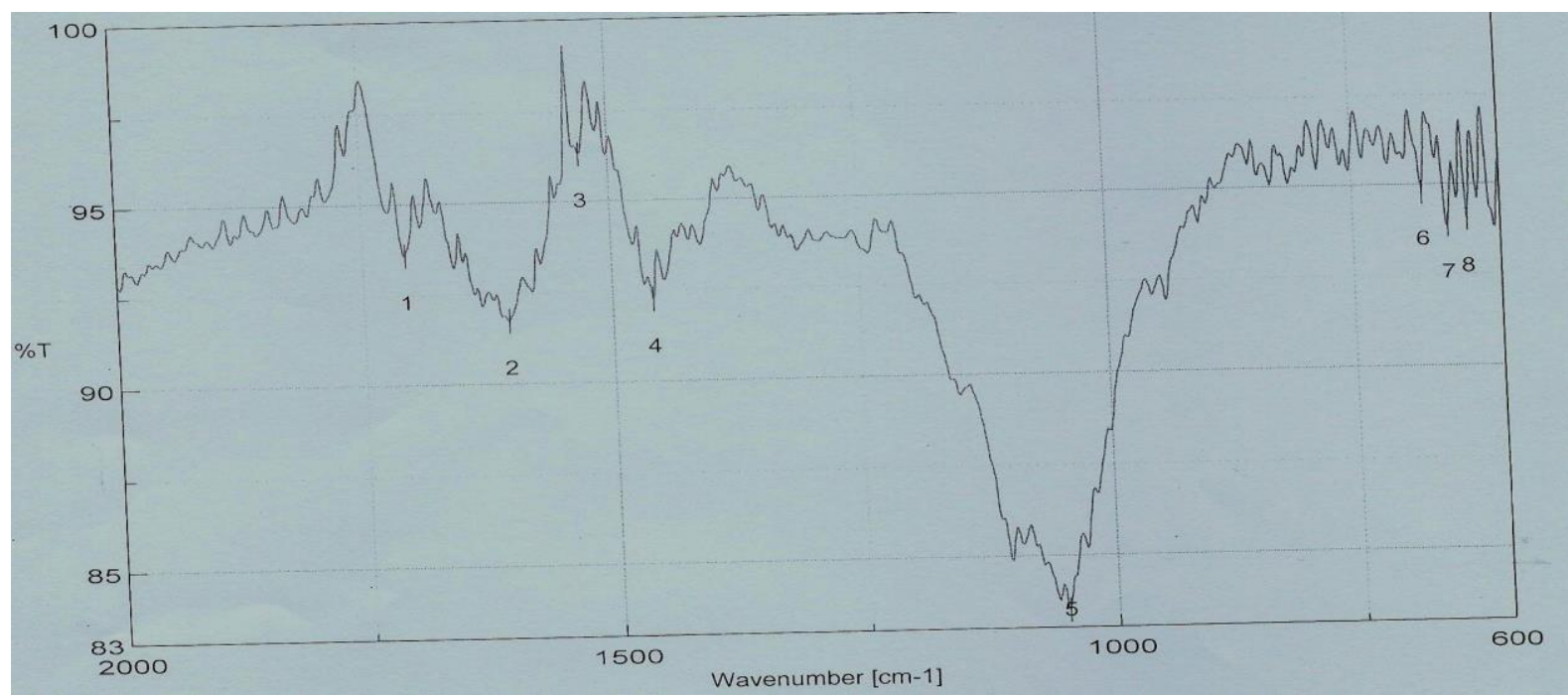

Figure 2: IR spectrum of Valsartan and SR excipients

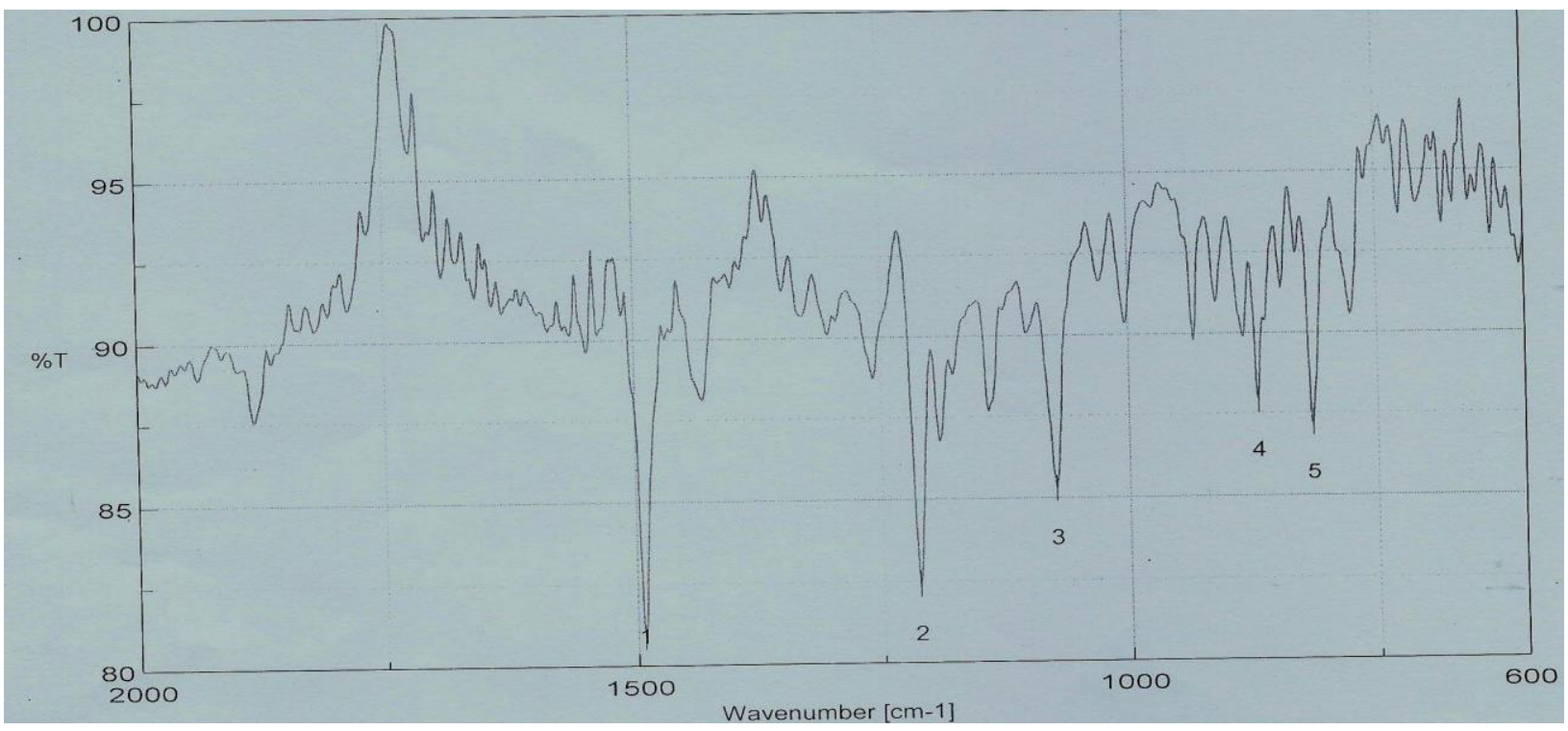

Figure 3: IR spectrum of Nebivolol

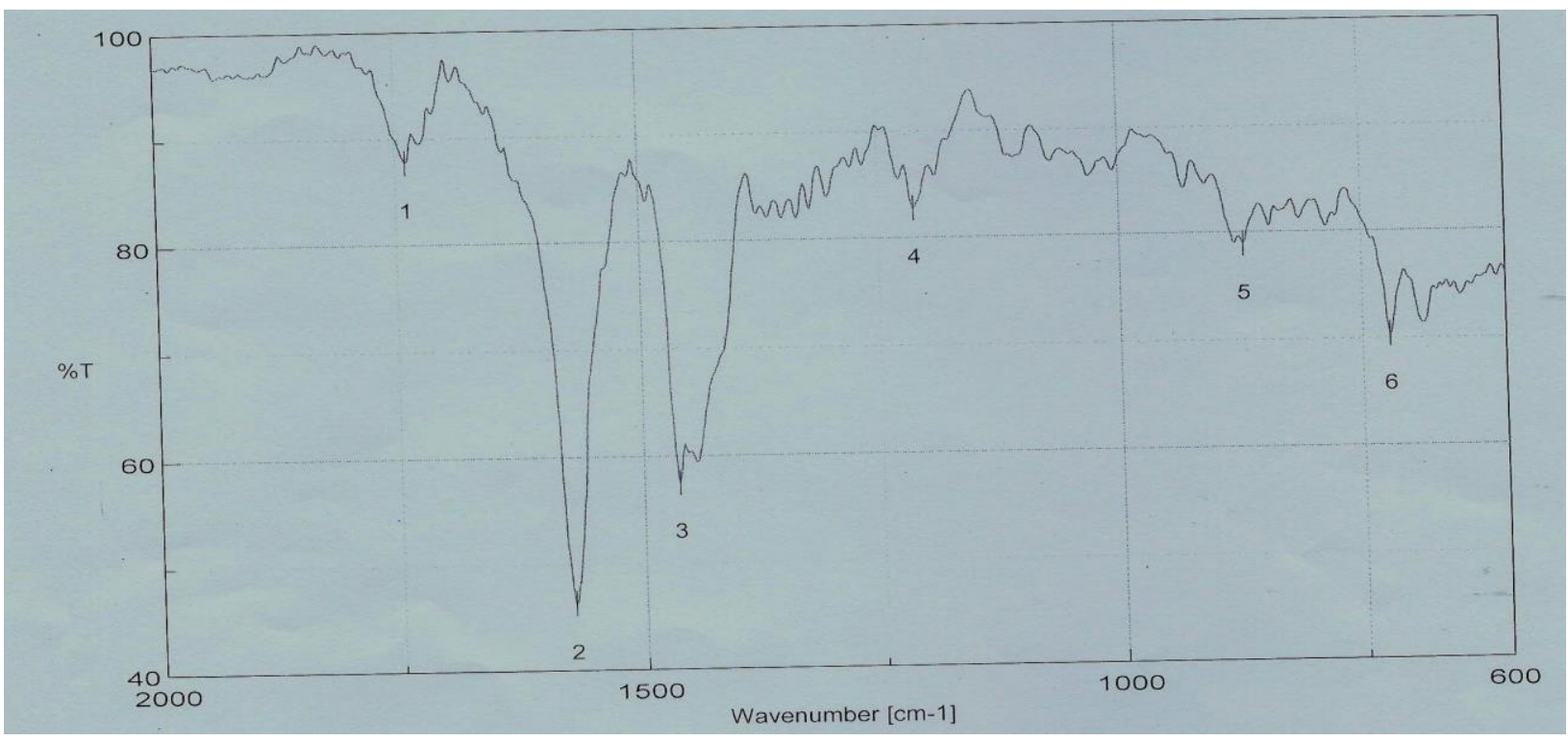

Figure 4: IR spectrum of Nebivolol and IR excipients 
Table 1: Excipient compatibility studies for Valsartan

\begin{tabular}{|c|c|c|c|c|c|c|c|}
\hline \multirow{3}{*}{ S.N. } & \multirow{3}{*}{ Drug+ Excipients } & \multirow{3}{*}{ Ratio } & \multirow{3}{*}{ Initial } & \multicolumn{4}{|c|}{ Condition } \\
\hline & & & & \multicolumn{4}{|c|}{$400 \mathrm{C} / 75 \% \mathrm{RH}$} \\
\hline & & & & 7 days & 14 days & 30 days & Conclusion \\
\hline 1. & $\begin{array}{l}\text { Valsartan + Microcrystalline cellulose } \\
\text { (Avicel PH 102) }\end{array}$ & $1: 1$ & \multirow{6}{*}{$\begin{array}{c}\text { A white } \\
\text { or almost } \\
\text { white } \\
\text { crystalline } \\
\text { powder }\end{array}$} & $\begin{array}{c}\text { No } \\
\text { change }\end{array}$ & No change & No change & compatible \\
\hline 2. & Valsartan + Dibasic calcium Phosphate & $1: 1$ & & $\begin{array}{c}\text { No } \\
\text { change }\end{array}$ & No change & No change & compatible \\
\hline 3. & $\begin{array}{l}\text { Valsartan +Hydroxy Propyl Methyl } \\
\text { Cellulose (Metolose 90SH 4000SR) }\end{array}$ & $1: 1$ & & $\begin{array}{c}\text { No } \\
\text { change }\end{array}$ & No change & No change & compatible \\
\hline 4. & $\begin{array}{l}\text { Valsartan + Hydroxy Propyl Methyl } \\
\text { Cellulose (HPMC K100M) }\end{array}$ & $1: 1$ & & $\begin{array}{c}\text { No } \\
\text { change }\end{array}$ & No change & No change & compatible \\
\hline 5. & $\begin{array}{l}\text { Valsartan + Colloidal silicon Dioxide } \\
\text { (Aerosil-200) }\end{array}$ & $1: 0.25$ & & $\begin{array}{c}\text { No } \\
\text { change }\end{array}$ & No change & No change & compatible \\
\hline 6. & Valsartan + Magnesium Stearate & $1: 0.25$ & & $\begin{array}{c}\text { No } \\
\text { change }\end{array}$ & No change & No change & compatible \\
\hline
\end{tabular}

Table 2: Excipient compatibility studies for Nebivolol Hydrochloride

\begin{tabular}{|c|c|c|c|c|c|c|c|}
\hline \multirow{3}{*}{ S.N. } & \multirow{3}{*}{ Drug+ Excipients } & \multirow{3}{*}{ Ratio } & \multirow{3}{*}{ Initial } & \multirow{2}{*}{\multicolumn{4}{|c|}{$\begin{array}{c}\text { Condition } \\
\text { 400C } / 75 \% \mathrm{RH}\end{array}$}} \\
\hline & & & & & & & \\
\hline & & & & 7 days & 14 days & 30 days & Conclusion \\
\hline 1. & $\begin{array}{l}\text { Nebivolol Hydrochloride + Dibasic } \\
\text { calcium Phosphate }\end{array}$ & $1: 1$ & \multirow{8}{*}{$\begin{array}{l}\text { A white or } \\
\text { almost white } \\
\text { crystalline } \\
\text { powder }\end{array}$} & $\begin{array}{c}\text { No } \\
\text { change }\end{array}$ & $\begin{array}{c}\text { No } \\
\text { change }\end{array}$ & $\begin{array}{c}\text { No } \\
\text { change }\end{array}$ & Compatible \\
\hline 2. & $\begin{array}{c}\text { Nebivolol Hydrochloride + } \\
\text { Microcrystalline Cellulose (Avicel PH } \\
\text { 102) }\end{array}$ & $1: 1$ & & $\begin{array}{l}\text { No } \\
\text { change }\end{array}$ & $\begin{array}{l}\text { No } \\
\text { change }\end{array}$ & $\begin{array}{l}\text { No } \\
\text { change }\end{array}$ & Compatible \\
\hline 3 & $\begin{array}{l}\text { Nebivolol Hydrochloride + Sodium } \\
\text { Starch Glycolate }\end{array}$ & $1: 1$ & & $\begin{array}{l}\text { No } \\
\text { change }\end{array}$ & $\begin{array}{l}\text { No } \\
\text { change }\end{array}$ & $\begin{array}{l}\text { No } \\
\text { change }\end{array}$ & Compatible \\
\hline 4 & $\begin{array}{l}\text { Nebivolol Hydrochloride + } \\
\text { Croscarmellose sodium }\end{array}$ & $1: 1$ & & $\begin{array}{l}\text { No } \\
\text { change }\end{array}$ & $\begin{array}{l}\text { No } \\
\text { change }\end{array}$ & $\begin{array}{l}\text { No } \\
\text { change }\end{array}$ & Compatible \\
\hline 5 & $\begin{array}{c}\text { Nebivolol Hydrochloride + Hydroxy } \\
\text { Propyl Methyl Cellulose ( HPMC } \\
\text { K100M) }\end{array}$ & $1: 10$ & & $\begin{array}{l}\text { No } \\
\text { change }\end{array}$ & $\begin{array}{l}\text { No } \\
\text { change }\end{array}$ & $\begin{array}{l}\text { No } \\
\text { change }\end{array}$ & Compatible \\
\hline 6 & $\begin{array}{c}\text { Nebivolol Hydrochloride + Hydroxy } \\
\text { Propyl Methyl Cellulose (Metolose } \\
\text { aمsu } 10 \Omega 0 \mathrm{CD} \text { ) }\end{array}$ & $1: 10$ & & $\begin{array}{l}\text { No } \\
\text { change }\end{array}$ & $\begin{array}{l}\text { No } \\
\text { change }\end{array}$ & $\begin{array}{l}\text { No } \\
\text { change }\end{array}$ & Compatible \\
\hline 7 & $\begin{array}{l}\text { Nebivolol Hydrochloride }+ \text { Colloidal } \\
\text { silicon Dioxide (Aerosil-200) }\end{array}$ & $1: 0.25$ & & $\begin{array}{l}\text { No } \\
\text { change }\end{array}$ & $\begin{array}{l}\text { No } \\
\text { change }\end{array}$ & $\begin{array}{l}\text { No } \\
\text { change }\end{array}$ & Compatible \\
\hline 8 & $\begin{array}{l}\text { Nebivolol Hydrochloride + } \\
\text { Magnesium Stearate }\end{array}$ & $1: 0.25$ & & $\begin{array}{l}\text { No } \\
\text { change }\end{array}$ & $\begin{array}{l}\text { No } \\
\text { change }\end{array}$ & $\begin{array}{l}\text { No } \\
\text { change }\end{array}$ & Compatible \\
\hline 9 & $\begin{array}{l}\text { Nebivolol Hydrochloride + FD\&C } \\
\text { blueLake }\end{array}$ & $1: 0.25$ & $\begin{array}{l}\text { A yellow } \\
\text { crystalline } \\
\text { powder }\end{array}$ & $\begin{array}{l}\text { No } \\
\text { change }\end{array}$ & $\begin{array}{l}\text { No } \\
\text { change }\end{array}$ & $\begin{array}{l}\text { No } \\
\text { change }\end{array}$ & Compatible \\
\hline
\end{tabular}

Table 3: Pre-compression Parameters of Nebivolol hydrochloride

\begin{tabular}{|c|c|c|c|c|c|}
\hline Formulation & $\begin{array}{c}\text { Angle of } \\
\text { Repose* }\end{array}$ & Bulk Density* & Tapped Density* & $\begin{array}{c}\text { Carr's. Index } \\
(\%)^{*}\end{array}$ & $\begin{array}{c}\text { Hausner's } \\
\text { Ratio* }^{*}\end{array}$ \\
\hline B1 & $280.46^{\prime} \pm 0.26$ & $0.6130 \pm 0.0071$ & $0.799 \pm 0.0021$ & $23.28 \pm 0.71$ & $1.304 \pm 0.008$ \\
\hline B2 & $280.46^{\prime} \pm 0.35$ & $0.613 \pm 0.0071$ & $0.799 \pm 0.0024$ & $23.28 \pm 0.75$ & $1.304 \pm 0.007$ \\
\hline B3 & $280.46^{\prime} \pm 0.72$ & $0.613 \pm 0.091$ & $0.799 \mathrm{v} 0.0019$ & $23.28 \pm 0.80$ & $1.304 \pm 0.098$ \\
\hline B4 & $270.23^{\prime} \pm 0.27$ & $0.597 \pm 0.0091$ & $0.789 \pm 0.0030$ & $23.94 \pm 0.69$ & $1.315 \pm 0.009$ \\
\hline B5 & $270.23^{\prime} \pm 0.34$ & $0.597 \pm 0.0092$ & $0.789 \pm 0.0023$ & $23.94 \pm 0.73$ & $1.315 \pm 0.089$ \\
\hline B6 & $270.23^{\prime} \pm 0.70$ & $0.597 \pm 0.0074$ & $0.789 \pm 0.0018$ & $23.94 \pm 0.79$ & $1.315 \pm 0.097$ \\
\hline
\end{tabular}

Where, *All values are mean $\pm S D, n=3$, 
Table 4: Pre-compression Parameters of Valsartan

\begin{tabular}{|c|c|c|c|c|c|}
\hline Formulation & Angle of Repose* & Bulk Density* & Tapped Density* & C. Index (\%)* & Hausner's Ratio* $^{*}$ \\
\hline B1 & $270.28^{\prime} \pm 0.27$ & $0.329 \pm 0.0094$ & $0.5280 \pm 0.0019$ & $37.73 \pm 0.77$ & $1.606 \pm 0.007$ \\
\hline B2 & $280.10^{\prime} \pm 0.71$ & $0.335 \pm 0.0075$ & $0.533 \pm 0.0025$ & $37.25 \pm 0.74$ & $1.594 \pm 0.006$ \\
\hline B3 & $280.35^{\prime} \pm 0.77$ & $0.305 \pm 0.008$ & $0.536 \pm 0.0028$ & $38.137 \pm 0.81$ & $1.759 \pm 0.097$ \\
\hline B4 & $280.32^{\prime} \pm 0.49$ & $0.350 \pm 0.009$ & $0.534 \pm 0.0026$ & $34.42 \pm 0.79$ & $1.525 \pm 0.078$ \\
\hline B5 & $280.36^{\prime} \pm 0.25$ & $0.333 \pm 0.0069$ & $0.522 \pm 0.0024$ & $36.26 \pm 0.82$ & $1.569 \pm 0.008$ \\
\hline B6 & $280.56^{\prime} \pm 0.78$ & $0.306 \pm 0.0077$ & $0.486 \pm 0.0029$ & $36.98 \pm 0.74$ & $1.587 \pm 0.087$ \\
\hline
\end{tabular}

Where, *All values are mean $\pm S D, n=3$,

Six formulations of bilayer tablets were prepared with the variation of excipients as mentioned Table No 5. Various steps (Sifting, Dry mixing, Prelubrication and Lubrication) involved in direct compression process. ${ }^{11}$ the compressed tablets were analyzed for post compression parameters. The details of the results are shown in table no 6.

Preparation of Nebivolol Hydrochloride immediate release (IR) layer:

The active Ingredient Nebivolol hydrochloride and diluents Micro crystalline cellulose (Avicel PH 102), Dibasic calcium Phosphate (anhydrous, FCC), were sifted through 40 mesh sieve. Sodium Starch Glycolate(731713H), Croscarmellose sodium (Ac di sol NF),were sifted through 60 mesh sieve. FD\&C blue lake, was sifted through 100 mesh sieve.

Dry mixing: Mix geometrically Nebivolol hydrochloride with Micro crystalline cellulose (Avicel pH 102), Dibasic calcium Phosphate, Disintegrants (SSG or CCS) and FD\&C blue lake, were taken in a poly bag and mixed for 15 minutes to ensure uniform mixing of the ingredients with the drug.

Preparation of Valsartan sustained release (SR) layer:

The active ingredient Valsartan, was passed through the 40 mesh sieve followed by the other ingredients were passed the same sieve.

Dry mixing: Valsartan, Polymer [Hydroxypropyl Methylcellulose K100M (Methocel K100M premium), K4M
(Methocel K4M premium)] diluents [Dibasic calcium Phosphate (anhydrous, FCC)] were taken in a planetary mixer and mixed for 15 minutes to ensure uniform mixing of the ingredients with the drug.

Prelubrication: Colloidal slicondioxide (Aerosil-200), sifted through 40 mesh sieve were mixed with dry mixed blend for 5 minutes.

Lubrication and compression of Bilayer tablets:

Magnesium stearate, was weighed and were passed through 60 mesh sieve. Then mixed with dried granules of Valsartan and Nebivolol Hydrochloride separately in a polybag for 5 minutes to get a uniform blend. Then the lubricated granules of Valsartan and Nebivolol Hydrochloride were added in the separate hopper in double rotary punching machine and compressed into Bilayer tablets using 13.5X.7.5mm oblong shape punches, at target weight of 300 $\mathrm{mg}$.

The stock standard solution of Nebivolol and Valsartan were prepared by dissolving accurate amount of the drugs initially in methanol and diluted with $6.8 \mathrm{ph}$. phosphate buffer to get the first stock solution. From this stock solution series of dilutions were made to get a concentration range of 5-100 ppm. A linearity graph was obtained by measuring the absorbance of the series of dilution of the drugs at $281 \mathrm{~nm}$ and $251 \mathrm{~nm}$ for Nebivolol hydrochloride and valsartan respectively.

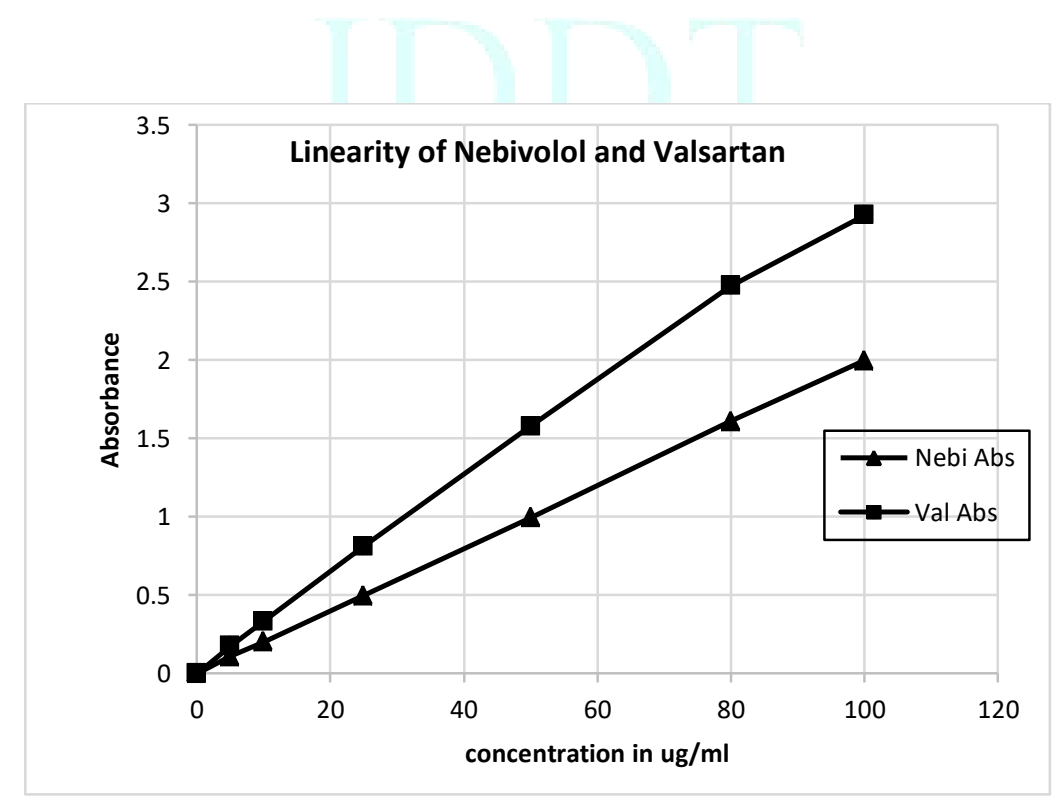

Figure 5: Linearity of Nebivolol Hydrochloride and Valsartan 
Table 5: Composition of Nebivolol Hydrochloride and Valsartan in IR layer and SR layer

Nebivolol Hydrochloride and Valsartan Bilayer tablets

Formulation Plan

\begin{tabular}{|c|c|c|c|c|c|c|c|c|c|c|c|c|c|}
\hline \multirow{2}{*}{$\begin{array}{l}\text { S. } \\
\text { N. }\end{array}$} & \multirow[b]{2}{*}{ Ingredients } & \multicolumn{2}{|c|}{ B1 } & \multicolumn{2}{|c|}{ B2 } & \multicolumn{2}{|c|}{ B3 } & \multicolumn{2}{|c|}{ B4 } & \multicolumn{2}{|c|}{ B5 } & \multicolumn{2}{|c|}{ B6 } \\
\hline & & $\underset{\mathrm{ab}}{\mathrm{mg} / \mathrm{T}}$ & $\begin{array}{c}\% \mathrm{~W} / \\
\mathrm{W}\end{array}$ & $\begin{array}{c}\mathrm{mg} / \mathrm{T} \\
\mathrm{ab}\end{array}$ & $\begin{array}{c}\% \mathrm{~W} / \\
\mathrm{W}\end{array}$ & $\begin{array}{c}\mathrm{mg} / \mathrm{T} \\
\mathrm{ab}\end{array}$ & $\begin{array}{c}\% \mathrm{~W} / \\
\mathrm{W}\end{array}$ & $\begin{array}{c}\mathrm{mg} / \mathrm{T} \\
\mathrm{ab}\end{array}$ & $\begin{array}{c}\% \mathrm{~W} / \\
\mathrm{W}\end{array}$ & $\begin{array}{c}\mathrm{mg} / \mathrm{T} \\
\mathrm{ab}\end{array}$ & $\begin{array}{c}\% \mathrm{~W} / \\
\mathrm{W}\end{array}$ & $\underset{\mathrm{ab}}{\mathrm{mg} / \mathrm{T}}$ & $\begin{array}{c}\text { \%W/ } \\
\mathrm{W}\end{array}$ \\
\hline \multicolumn{14}{|c|}{ First layer } \\
\hline 1 & $\begin{array}{c}\text { Nebivolol } \\
\text { Hydrochloride }\end{array}$ & 5 & 5 & 5 & 5 & 5 & 5 & 5 & 5 & 5 & 5 & 5 & 5 \\
\hline 2 & $\begin{array}{c}\text { Microcrystalline } \\
\text { cellulose (Avicel PH } \\
102 \text { ) }\end{array}$ & 37.95 & 37.95 & 32.95 & 32.95 & 34.95 & 34.95 & 34.95 & 34.95 & 32.95 & 32.95 & 37.95 & 37.95 \\
\hline 3 & $\begin{array}{l}\text { Dibasic calcium } \\
\text { Phosphate }\end{array}$ & 51.8 & 51.8 & 51.8 & 51.8 & 51.8 & 51.8 & 51.8 & 51.8 & 51.8 & 51.8 & 51.8 & 51.8 \\
\hline 4 & $\begin{array}{l}\text { Sodium starch } \\
\text { Glycolate }\end{array}$ & 4 & 4 & 4 & 4 & 4 & 4 & 0 & 0 & 0 & 0 & 0 & 0 \\
\hline 5 & $\begin{array}{l}\text { Croscarmellose } \\
\text { sodium }\end{array}$ & 0 & 0 & 0 & 0 & 0 & 0 & 4 & 4 & 4 & 4 & 4 & 4 \\
\hline 6 & FD\&C blue lake & 0.25 & 0.25 & 0.25 & 0.25 & 0.25 & 0.25 & 0.25 & 0.25 & 0.25 & 0.25 & 0.25 & 0.25 \\
\hline 7 & Mg. Stearate & 1 & 1 & 1 & 1 & 1 & 1 & 1 & 1 & 1 & 1 & 1 & 1 \\
\hline & Total & 100 & 100 & 100 & 100 & 100 & 100 & 100 & 100 & 100 & 100 & 100 & 100 \\
\hline \multicolumn{14}{|c|}{ Second layer } \\
\hline 8 & valsartan & 60 & 23.75 & 60 & 23.75 & 60 & 23.75 & 60 & 23.75 & 60 & 23.75 & 60 & 23.75 \\
\hline 9 & $\begin{array}{c}\text { Microcrystalline } \\
\text { cellulose (Avicel PH } \\
102 \text { ) }\end{array}$ & 86 & 49.25 & 98 & 55.25 & 91 & 52.25 & 92 & 52.25 & 76 & 44.25 & 16 & 34.25 \\
\hline 10 & HPMC K100M & 50 & 25 & 38 & 19 & 44 & 22 & 0 & 0 & 0 & 0 & 0 & 0 \\
\hline 11 & HPMC K4M & 0 & 0 & 0 & 0 & 0 & 0 & 44 & 22 & 60 & 30 & 120 & 40 \\
\hline 12 & Sio2 & 2 & 1 & 2 & 1 & 2 & 1 & 2 & 1 & 2 & 1 & 2 & 1 \\
\hline 13 & Mg. Stearate & 2 & 1 & 2 & 1 & 2 & 1 & 2 & 1 & 2 & 1 & 2 & 1 \\
\hline & Total & 200 & 100 & 200 & 100 & 200 & 100 & 200 & 100 & 200 & 100 & 200 & 100 \\
\hline
\end{tabular}

The compressed tablets were evaluated for weight variation, content uniformity, hardness, thickness and drug content8. The results are shown in table no 6

Table 6: Physical characterizations of Bi-layer tablets.

\begin{tabular}{|c|c|c|c|c|c|c|}
\hline Batch & \multirow{2}{*}{$\begin{array}{c}\text { Avg. Wt. }(\mathrm{mg}) \\
\text { code }\end{array}$} & $\begin{array}{c}\text { Hardness*(kg/c } \\
\mathrm{m} 2)\end{array}$ & \multirow{2}{*}{ Thickness ${ }^{*} \mathrm{~mm}$} & \multicolumn{2}{|c|}{ Drug content (\%)* } & \multirow{2}{*}{ Friability (\%) } \\
\cline { 5 - 7 } B1 & $304.3 \pm 1.24$ & $17.3 \pm 4.31$ & $3.91 \pm 2.31$ & 91.27 & 95.65 & $0.58 \pm 2.36$ \\
\hline B2 & $296.0 \pm 2.72$ & $15.93 \pm 5.21$ & $3.89 \pm 2.35$ & 90.75 & 95.49 & $0.56 \pm 4.21$ \\
\hline B3 & $306.2 \pm 4.24$ & $17.34 \pm 6.32$ & $3.91 \pm 3.52$ & 91.27 & 95.65 & $0.54 \pm 1.23$ \\
\hline B4 & $303.2 \pm 3.21$ & $18.67 \pm 3.5$ & $3.92 \pm 4.12$ & 91.27 & 95.65 & $0.58 \pm 4.21$ \\
\hline B5 & $302.9 \pm 1.52$ & $17.77 \pm 2.31$ & $3.91 \pm 4.36$ & 90.75 & 95.65 & $0.55 \pm 2.36$ \\
\hline B6 & $304.2 \pm 3.23$ & $12.64 \pm 4.21$ & $4.13 \pm 2.42$ & 91.27 & 95.49 & $0.56 \pm 4.25$ \\
\hline
\end{tabular}

Where, ${ }^{*}$ All values are mean $\pm S D, n=3$,

\section{In vitro dissolution study 12}

The in vitro dissolution of Nebivolol Hydrochloride and Valsartan bilayer tablets were determined using USP XXIII (basket method) dissolution apparatus. The basket was allowed to rotate at a speed of $50 \mathrm{rpm}$ and temperature of 37 $\pm 0.5^{\circ} \mathrm{C}$ was maintained. The dissolution medium used was $900 \mathrm{ml}$ of $0.1 \mathrm{~N} \mathrm{HCl}(\mathrm{pH} \mathrm{1.2)}$ ) for the initial 2hours followed by study in simulated intestinal fluid Phosphate buffer solution
(pH 6.8). Aliquots $(5 \mathrm{ml})$ of sample were collected at predetermined time intervals $(1,4,8,20,24 \mathrm{hrs}$.) from the dissolution apparatus and it was replaced with equal volume of fresh dissolution medium. The aliquots withdrawn were filtered through $0.45 \mu \mathrm{m}$ millipore filters. The concentration of both the drugs in the dissolution media was estimated by UV spectrophotometric method at $281 \mathrm{~nm}$ and $251 \mathrm{~nm}$ for Nebivolol hydrochloride and Valsartan respectively. 
Table 7: Dissolution parameters

\begin{tabular}{|l|l|l|}
\hline & Nebivolol Hydrochloride & Valsartan \\
\hline Dissolution media & $0.01 \mathrm{~N} \mathrm{HCl}$ & $\mathrm{pH} 6.80$ Phosphate Buffer \\
\hline Volume & $900 \mathrm{ml}$ & $900 \mathrm{ml}$ \\
\hline Model & USP TYPE II Paddle & USP TYPE II Paddle \\
\hline Rpm & 50 & 50 \\
\hline Time of sampling & $15,30 \mathrm{Mins}$ & $1,4,8,20 \mathrm{Hrs}$ \\
\hline Temperature & $370 \mathrm{c}$ & $370 \mathrm{c}$ \\
\hline
\end{tabular}

Table 8: Drug Release from Valsartan sustained release (SR) layer

\begin{tabular}{|c|c|c|c|c|c|c|}
\hline & B1 & B2 & B3* & B4 $^{*}$ & B5* $^{*}$ & B6* $^{*}$ \\
\hline Time Hrs & \%CDR & \%CDR & \%CDR & \%CDR & \%CDR & \%CDR \\
\hline 0 & 0 & 0 & 0 & 0 & 0 & 0 \\
\hline 1 & $11 \pm 8.94$ & $12 \pm 7.45$ & $18 \pm 22.41$ & $11 \pm 0.00$ & $9 \pm 9.94$ & $10 \pm 0.00$ \\
\hline 4 & $25 \pm 11.50$ & $25 \pm 6.20$ & $30 \pm 1.36$ & $27 \pm 2.03$ & $24 \pm 16.81$ & $24 \pm 6.46$ \\
\hline 8 & $37 \pm 7.77$ & $39 \pm 8.70$ & $46 \pm 3.00$ & $43 \pm 4.01$ & $38 \pm 14.49$ & $40 \pm 2.58$ \\
\hline 20 & $64 \pm 5.17$ & $66 \pm 5.91$ & $80 \pm 5.92$ & $79 \pm 8.50$ & $70 \pm 13.66$ & $72 \pm 0.57$ \\
\hline 24 & $71 \pm 3.64$ & $76 \pm 5.92$ & $84 \pm 6.76$ & $87 \pm 9.33$ & $80 \pm 13.80$ & $81 \pm 2.92$ \\
\hline
\end{tabular}

Where, ${ }^{*}$ All values are mean $\pm \mathrm{RSD}, \mathrm{n}=6$. ,

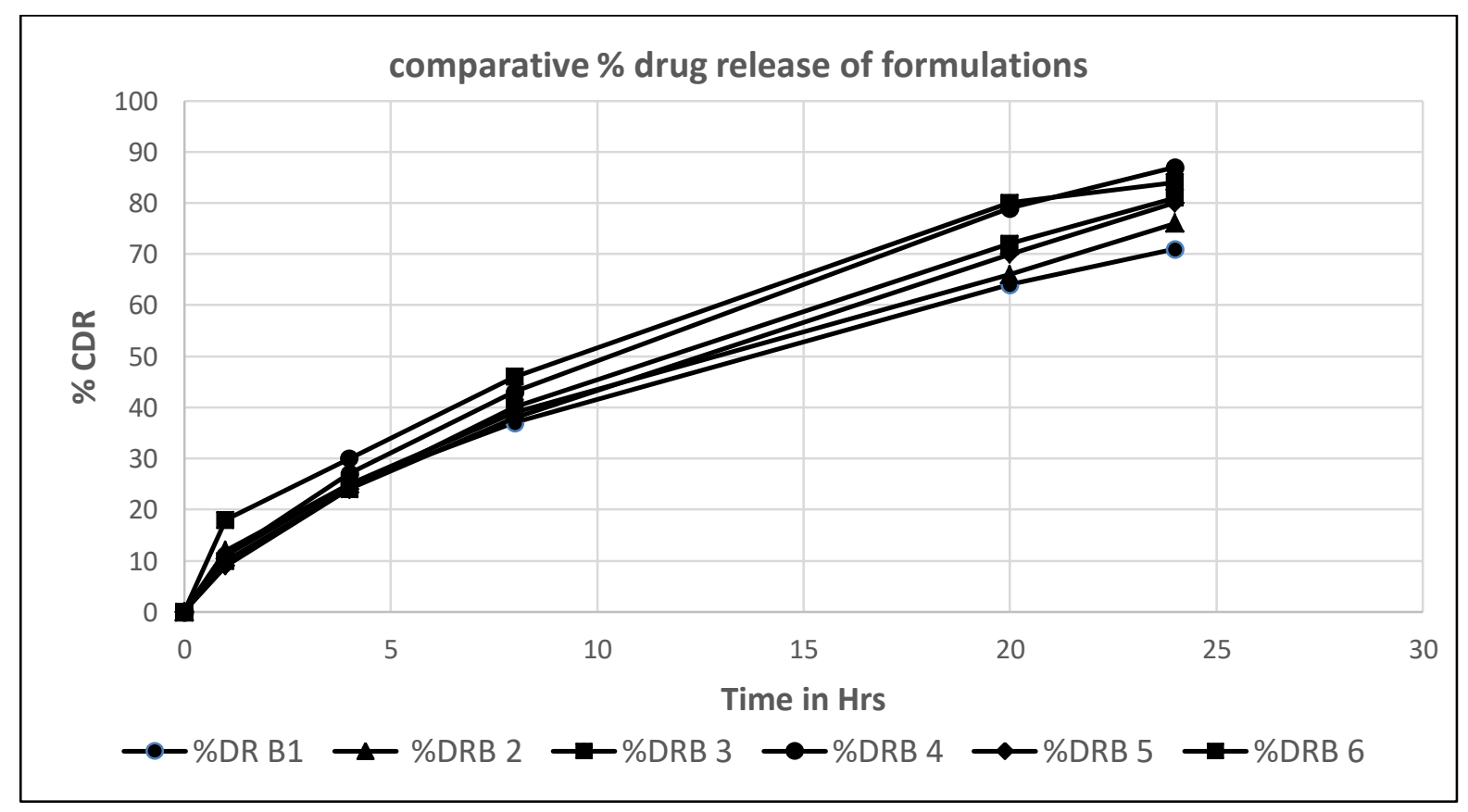

Figure 6: Drug Release from Valsartan SR layer for formulations B1 to B6

Table 9: Drug Release from Nebivolol hydrochloride immediate release (SR) layer

\begin{tabular}{|c|c|c|}
\hline \multirow{2}{*}{ I set Dissolution of IR1 with SR1,SR,SR3 } & \multicolumn{2}{|c|}{ Time mins } \\
\cline { 2 - 3 } & $65 \pm 3.94$ & $79 \pm 7.36$ \\
\hline B1* & $71 \pm 4.91$ & $83 \pm 1.77$ \\
\hline B2* & $79 \pm 6.72$ & $92 \pm 3.47$ \\
\hline B3* & & $83 \pm 6.17$ \\
\hline II set DIssolution of IR2with SR4,SR5,SR6 & $71 \pm 5.49$ & 835.25 \\
\hline B4* & $67 \pm 1.12$ & $81 \pm 4.73$ \\
\hline B5* & $74 \pm 22.31$ & \\
\hline B6* & & \\
\hline
\end{tabular}

Where, ${ }^{*}$ All values are mean $\pm \mathrm{RSD}, \mathrm{n}=6$. , 


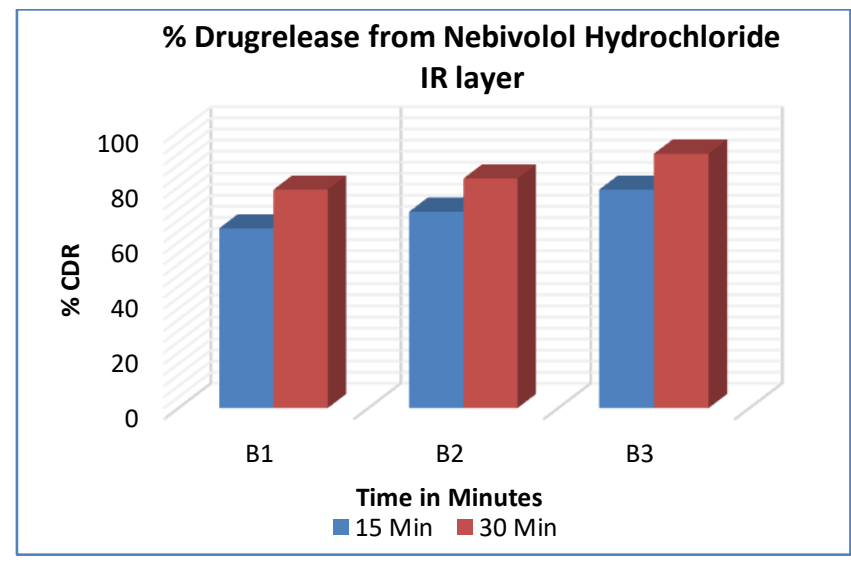

Figure 7: Drug Release from Nebivolol Hydrochloride IR layer for formulations B1 to B3

From the results of the evaluation formulation B3 was considered best formulation where the IR layer containing Nebivolol layer showing maximum release of $92 \%$ in 30 min

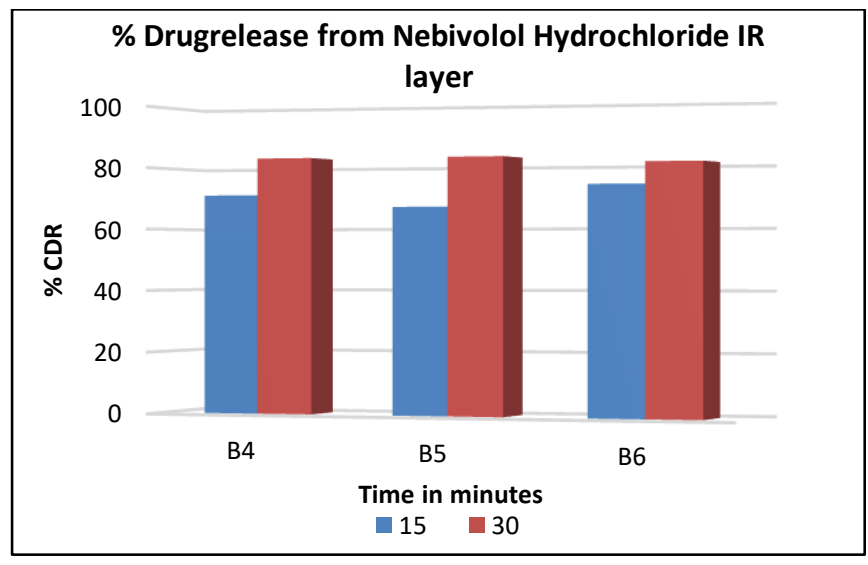

Figure 8: Drug Release from Nebivolol Hydrochloride IR layer for formulations B4 to B6

and SR layer containing Valsartan layer showing a slow and sustained release for a period of $24 \mathrm{hrs}$. B3 was subjected to curve fitting analysis to predict the pattern of drug release.

Table 10: Release kinetics

\begin{tabular}{|c|c|c|c|c|c|}
\hline Model & Zero order & Higuchi & $\begin{array}{c}\text { Korsemeyer } \\
\text { Peppas }\end{array}$ & $\begin{array}{c}\text { Hixon } \\
\text { Crowell }\end{array}$ & First order \\
\hline $\begin{array}{c}\text { Correlation } \\
\text { coefficient r }\end{array}$ & 0.9746495 & 0.9971163 & 0.8165266 & 0.058066 & -0.894879 \\
\hline
\end{tabular}

\section{Interpretation of the kinetic studies:}

- The $r$ value for zero order release indicates the SR layer containing valsartan follows zero order release pattern where in the drug release at any moment is independent of the initial concentration.

- The $r$ value for Higuchi model infers the SR layer follows a drug release by diffusion process based on Fickian law of diffusion.

- $\quad$ The $r$ value for Peppas model shows no linearity.

- The $r$ value for Hixon Crowell value indicates no linearity and hence proves that the drug release from SR layer is not by dissolution.

\section{In vivo pharmacokinetic studies: 13}

An in vivo pharmacokinetic study was carried out according to ethical guidelines for investigations in laboratory animals and approved by the Institutional Animal Ethics Committee (IAEC) reference No. in vivo/041.All procedures and care of the rabbits were in accordance with institutional guidelines for animal use in research. Four healthy male albino rabbit weighing 2-2.5 were fasted overnight. Tablets were administered orally. Rabbits were held in rabbit restrainers during blood sampling. Blood samples were collected from marginal ear veins at predetermined intervals of $0,0.5,1,2$, $3,4,6,8,20$ and $24 \mathrm{~h}$ post dose into heparinized tubes. Plasma samples were obtained following centrifugation of blood and kept frozen at -20 degree until analysis until liquid chromatography/mass spectrometry (LC/MS-MS) analysis.

Table 11: Plasma concentration of Nebivolol Hydrochloride in rabbit plasma

\begin{tabular}{|c|c|c|c|c|c|}
\hline $\begin{array}{c}\text { Animal } \\
\text { code }\end{array}$ & 1 & 2 & 3 & 4 & avg conc \\
\hline time (hrs) & conc ng/mL & conc ng/mL & conc ng/mL & conc ng/mL & ng/ml \\
\hline 0 & 0 & 0 & 0 & 0 & 0 \\
\hline 0.5 & 72.1 & 32.9 & 18 & 22.5 & 36.4 \\
\hline 1 & 17.7 & 25.4 & 22.6 & 22.1 & 22 \\
\hline 2 & 14.7 & 19.4 & 24 & 23.6 & 20.4 \\
\hline 3 & 221 & 61.3 & 61 & 41.9 & 96.3 \\
\hline 4 & 31.6 & 72.4 & 34.1 & 35.9 & 43.5 \\
\hline 6 & 65.5 & 14.8 & 23.2 & 24.3 & 32 \\
\hline 8 & 18.4 & 30.1 & 49.2 & 34.1 & 33 \\
\hline 20 & 31.9 & 27.7 & 25.4 & 23.9 & 27.2 \\
\hline 24 & 20.1 & 23.4 & 40.1 & 18.5 & 25.5 \\
\hline
\end{tabular}


Table 12: Plasma concentration of Valsartan in rabbit plasma

\begin{tabular}{|c|c|c|c|c|c|}
\hline Animal code & 1 & 2 & 3 & 4 & avg conc \\
\hline Time (hrs) & conc ng/mL & conc ng/mL & conc ng/mL & conc ng/mL & conc ng/mL \\
\hline 0 & 0 & 0 & 0 & 0 & 0 \\
\hline 0.5 & 12600 & 15600 & 6870 & 5020 & 10022.5 \\
\hline 1 & 8600 & 12200 & 3400 & 4190 & 7097.5 \\
\hline 2 & 15900 & 9980 & 1450 & 4980 & 8077.5 \\
\hline 3 & 17700 & 3100 & 3090 & 13400 & 9322.5 \\
\hline 4 & 12100 & 9970 & 26100 & 1570 & 6562.5 \\
\hline 6 & 19400 & 6770 & 2410 & 3760 & 8085 \\
\hline 8 & 1610 & 1210 & 4620 & 537 & 1994.3 \\
\hline 20 & 20500 & 24400 & 30400 & 34900 & 27550 \\
\hline 24 & 21100 & 24200 & 24300 & 21900 & 22875 \\
\hline
\end{tabular}

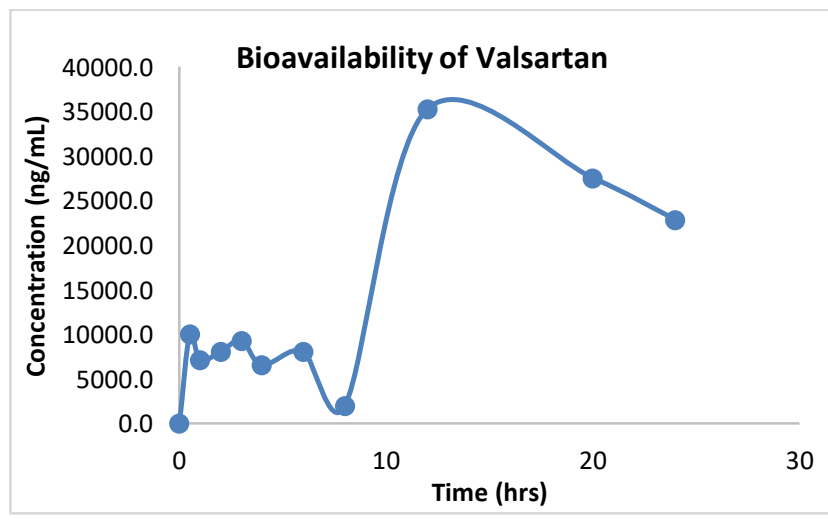

Figure 9: Bioavailability of Valsartan

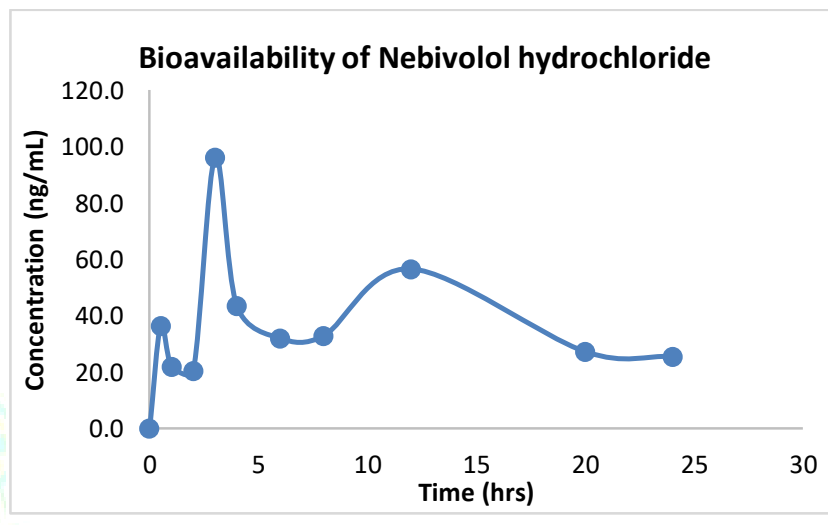

Figure 10: Bioavailability of Nebivolol Hydrochloride

Table 13: Details of LCMS analysis

\begin{tabular}{|c|c|c|c|c|c|}
\hline \multicolumn{6}{|c|}{ LC conditions } \\
\hline Column & \multicolumn{5}{|c|}{ ACE C18 PFP (150 x 4.6mm, $3 \mu$ particle size) } \\
\hline Flow rate & \multicolumn{5}{|c|}{$1 \mathrm{ml} / \mathrm{min}$} \\
\hline Column oven Temp. & \multicolumn{5}{|l|}{$40^{\circ} \mathrm{C}$} \\
\hline Injection volume & \multicolumn{5}{|l|}{$10 \mu \mathrm{l}$} \\
\hline \multirow[t]{7}{*}{ Mobile phase } & \multicolumn{5}{|c|}{$\begin{array}{l}\text { A-----0.1\% formic acid in water } \\
\text { B-----Acetonitrile+0.1\% formic acid } \\
\text { A:B-gradient elution }\end{array}$} \\
\hline & Mins & B.Conc. & & & \\
\hline & 0.01 & $30 \%$ & & & \\
\hline & 5.00 & $30 \%$ & & & \\
\hline & 8.00 & $80 \%$ & & & \\
\hline & 10.00 & $30 \%$ & & & \\
\hline & 12.00 & $30 \%$ & & & \\
\hline \multicolumn{6}{|l|}{ MS/MS conditions } \\
\hline Source & \multicolumn{5}{|c|}{ Electron spray ionization (ESI $+\&-$ mode) } \\
\hline Source Temp. & \multicolumn{5}{|c|}{$420^{\circ} \mathrm{C}$} \\
\hline Curtain gas & \multicolumn{5}{|l|}{$30 \mathrm{psi}$} \\
\hline Ion source gas (GS1) & \multicolumn{5}{|l|}{$50 \mathrm{psi}$} \\
\hline Ion source gas (GS2) & \multicolumn{5}{|l|}{$60 \mathrm{psi}$} \\
\hline Scan time & \multicolumn{5}{|l|}{$100 \mathrm{msec}$} \\
\hline \multicolumn{6}{|l|}{ MRM details } \\
\hline Name of the analyte & Transition state & $\mathrm{CE}(\mathrm{V})$ & $\mathrm{DP}(\mathrm{V})$ & FP (V) & $\begin{array}{l}\text { Ion source } \\
\text { voltage }\end{array}$ \\
\hline Valsartan & $434.0>179.0$ & $-30 \mathrm{~V}$ & $-60 \mathrm{~V}$ & $-400 \mathrm{~V}$ & $-3500 \mathrm{~V}$ \\
\hline Nebivolol & $406.1>151.0$ & $40 \mathrm{~V}$ & $50 \mathrm{~V}$ & $400 \mathrm{~V}$ & $3500 \mathrm{~V}$ \\
\hline Phenacetin (Internal standard) & $180.05>110.05$ & $30 \mathrm{~V}$ & $20 \mathrm{~V}$ & $400 \mathrm{~V}$ & $3500 \mathrm{~V}$ \\
\hline
\end{tabular}

$\mathrm{CE}=$ Collision Energy; DP: Declustering Potential; FP = Focusing Potential 
An ionization was checked in both the ionization mode (Positive and negative mode) and it was observed that Valsartan was shown good ionization in negative mode (deprotonated) and Nebivolol and Phenacetin (IS) were given good intense response in positive ionization mode (protonated).

\section{Standard curve:}

A stock cocktail solution (containing Nebivolol and Valsartan) ranging from $1000-0.312 \mu \mathrm{g} / \mathrm{ml}$ was prepared using LC-MS grade methanol. Further this was diluted to obtain a working standard solution ranging from $25.9 \mathrm{ng} / \mathrm{ml}$ to $83000 \mathrm{ng} / \mathrm{ml}$. The dilution was performed by pipetting $33.2 \mu \mathrm{L}$ separately from each stock and making up the volume to $200 \mu \mathrm{L}$ using untreated plasma and mixed by vortex for 30 seconds. After 10 minutes of incubation, $10 \mu \mathrm{L}$ of (25ppm) Phenacetin (internal standard) was added and vortex for 30 seconds. $600 \mu \mathrm{L}$ of methanol was added to precipitate the proteins. The samples were subjected to centrifugation at $8000 \mathrm{rpm}$ for 5 minutes at $15^{\circ} \mathrm{C}$. The supernatant was separated and subjected to LC-MS/MS analysis.

\section{Procedure:}

To the assay vial, $200 \mu$ l of rabbit plasma was added followed by addition of $10 \mu \mathrm{l}$ of internal standard Phenacetin at the concentration of $25 \mathrm{ppm}$. $600 \mu \mathrm{l}$ of $0.1 \%$ formic acid in methanol was added to precipitate the plasma contents mixed by vortex for 30 seconds. The above samples were subjected to vortex for 30 seconds followed by centrifugation at $8000 \mathrm{rpm}$ for 5 minutes at $15^{\circ} \mathrm{C}$. The supernatant was collected and subjected to LCMS analysis. The pharmacokinetic parameters were estimated using Phoenix WinNonlin® (Version 8.1, USA).

Table 14: Pharmacokinetic parameters of Nebivolol Hydrochloride and valsartan in rabbits following single dose administration of Bilayer tablet

\begin{tabular}{|c|c|c|c|c|c|c|c|}
\hline \multirow[b]{3}{*}{ Animal ID } & & \multicolumn{6}{|c|}{ ANALYTE } \\
\hline & \multirow[b]{2}{*}{$\begin{array}{l}\text { Dose } \\
\text { (mg) }\end{array}$} & \multicolumn{3}{|c|}{ Nebivolol } & \multicolumn{3}{|c|}{ Valsartan } \\
\hline & & $\begin{array}{l}\text { Tmax } \\
\text { (hr) }\end{array}$ & $\begin{array}{l}\mathrm{cmax} \\
\mathrm{ng} / \mathrm{ml}\end{array}$ & $\begin{array}{l}\text { AUC last } \\
\text { hr*ng/ml }\end{array}$ & $\operatorname{Tmax}(\mathrm{hr})$ & $\begin{array}{l}\mathrm{cmax} \\
\mathrm{ng} / \mathrm{ml}\end{array}$ & $\begin{array}{c}\text { AUC last } \\
\mathrm{hr}^{*} \mathrm{ng} / \mathrm{ml}\end{array}$ \\
\hline 1 & (5/60mg) & 3 & 221 & 766.37 & 24 & 21000 & 313814.1 \\
\hline 2 & (5/60mg) & 4 & 72.4 & 693.44 & 20 & 24400 & 303544 \\
\hline 3 & (5/60mg) & 3 & 61 & 814.98 & 20 & 30400 & 369320.6 \\
\hline 4 & (5/60mg) & 3 & 41.9 & 637.24 & 20 & 34900 & 356605.2 \\
\hline 5 & Mean & 3.25 & 99.075 & 728.009 & 21 & 27700 & 335821.1 \\
\hline 6 & SD & 0.5 & 82.252 & 78.464 & 2 & 6153.048 & 32043.09 \\
\hline 7 & SE & 0.25 & 41.26 & 39.232 & 1 & 3076.524 & 16021.55 \\
\hline 8 & CV\% & 15.4 & 83 & 10.8 & 9.5 & 22.2 & 9.5 \\
\hline 9 & Min & 3 & 41.9 & 637.24 & 20 & 21100 & 303544.5 \\
\hline 10 & Median & 3 & 66.7 & 729.91 & 20 & 27400 & 335209.7 \\
\hline 11 & Max & 4 & 221 & 814.98 & 24 & 34900 & 369320.6 \\
\hline
\end{tabular}

\section{In vitro In vivo correlation (IVIVC)}

Recently, application of IVIVC has been suggested in the pharmaceutical field for development of novel formulations to reduce the time and cost of manufacturing. ${ }^{14}$ The FDA has developed a regulatory guidance for both immediate and modified release dosage forms, thus minimizing the need for bioavailability studies as a part of the formulation design and optimization process. Among the poorly water soluble drugs, BCS class II drugs could be applied to IVIVC, since dissolution is the rate limiting step for drug absorption in gut. ${ }^{15}$ This concept holds good for Nebivolol Hydrochloride which belong to BCS class II, also an immediate release layer of this drug would further enhance the dissolution there by improving absorption. Though Valsartan belongs to BCS class IV, it behaves as BCS class III drug since it shows good solubility in intestinal $\mathrm{Ph}$. hence can be considered for IVIVC.
Table 15: IVIVC for Valsartan

\begin{tabular}{|c|c|c|}
\hline Time $(\mathrm{hr})$ & $\begin{array}{c}\text { In-vivo } \\
\text { Plasma conc ng/ml }\end{array}$ & $\begin{array}{c}\text { In-vitro } \\
\text { \%CDR }\end{array}$ \\
\hline 0 & 0 & 0 \\
\hline 0.5 & 10022.5 & - \\
\hline 1 & 7097.5 & 18 \\
\hline 2 & 8077.5 & - \\
\hline 3 & 9322.5 & - \\
\hline 4 & 6562.5 & 30 \\
\hline 6 & 8085 & - \\
\hline 8 & 1994.3 & 46 \\
\hline 20 & 27550 & 80 \\
\hline 24 & 22875 & 84 \\
\hline
\end{tabular}




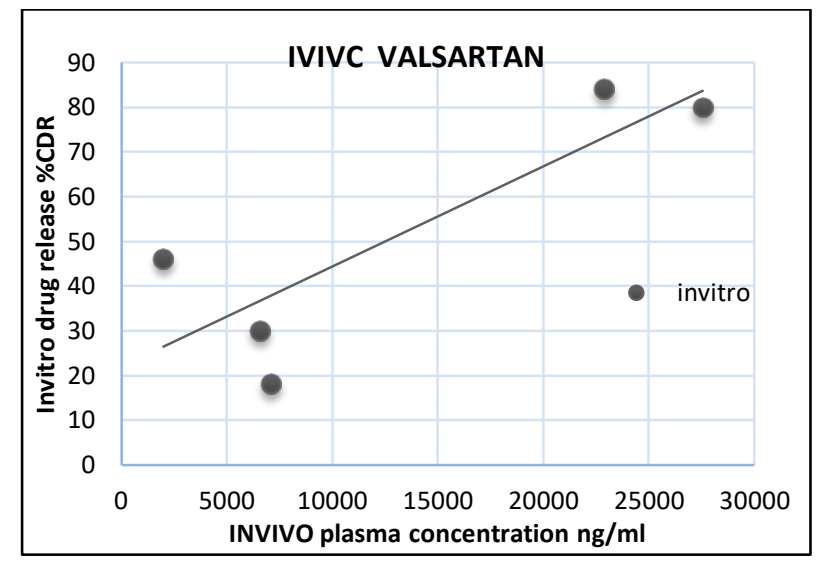

Figure 11: IVIVC for Valsartan $r=0.852475$

Table 16: IVIVC for Nebivolol Hydrochloride

\begin{tabular}{|c|c|c|}
\hline Time (hr) & $\begin{array}{c}\text { Invivo } \\
\text { Plasma conc } \\
\text { ng/ml }\end{array}$ & $\begin{array}{c}\text { invitro } \\
\text { \%CDR }\end{array}$ \\
\hline 0 & 0 & 0 \\
\hline 0.25 & 36.4 & 79 \\
\hline 0.5 & 22 & 92 \\
\hline 1 & 20.4 & - \\
\hline 2 & 96.3 & - \\
\hline
\end{tabular}

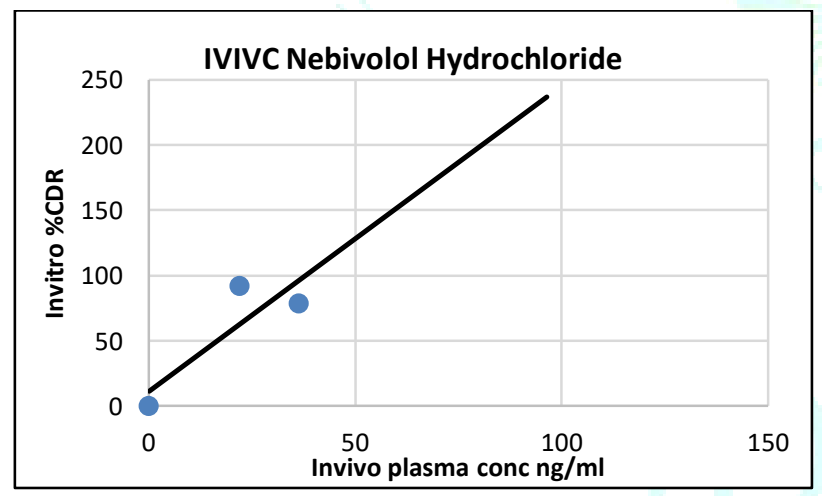

Figure 12: IVIVC of Nebivolol Hydrochloride $r=0.860495$

\section{Stability studies 16}

In the present analysis, stability studies were carried out for both at room temperature and accelerated stability conditions. The conditions for storing at room temperature were kept as $30 \pm 2{ }^{\circ} \mathrm{C}$ and $65 \pm 5 \% \mathrm{RH}$ and for accelerated stability conditions were kept at $40 \pm 2^{\circ} \mathrm{C}$ and $75 \pm 5 \% \mathrm{RH}$ in a humidity chamber. At regular intervals of time $(0,2,4$ and 6 months) samples were withdrawn and evaluated for drug content and in-vitro release profile. All the parameters were within the acceptable limits.

\section{RESULTS AND DISCUSSION}

Various formulations of Bilayer tablets were prepared and evaluated with an aim to develop Valsartan as sustained release layer and Nebivolol Hydrochloride as an immediate release layer for improving hypertension therapy of two drugs and also patient's compliance. To minimize the critical process parameters, direct compression method was selected for the formulation. The polymers and other excipients were selected based on the satisfying results produced during Drug-Excipients compatibility studies to develop the final formulation (Table 1\&2). Results show the compatibility of drug with the excipients. The physicochemical evaluation results for the granules of all trials pass the official limits with respect bulk and tapped density, angle of repose, Carr's index ans hausners' ratio (Table 3). In present work a bulk density in the range of 0.5 $0.6 \mathrm{gm} / \mathrm{cm} 3$ indicates a good packing characteristics. The Carr's compressibility index was found to be in the range of $23 \%$ which suggested optimal compressibility. The values of hausner ratio where found in the range of 1.30 suggested optimal flowability of powder blend. The angle of repose of all the blend was within range of 28 to 28 indicated excellent flow property of powder blend. The bilayer tablets were evaluated for different physical parameter. The hardness of bilayer tablet was found in the range of 12 to $17 \mathrm{~kg} / \mathrm{cm} 2$ which was more as compare to individual layer because of double compression. The thickness of the bilayer tablet was in the range of $3-4 \mathrm{~mm}$ which is an excellent value for double layer. The friability was $0.54-0.58 \%$ for bilayer tablet which was less than 1 indicating good handling of tablet. The weight variation study showed low standard deviation uniformity in weight of the tablets $300 \pm 0.06 \mathrm{mg} .{ }^{17}$ In the formula of Nebivolol Hydrochlioride immediate release layer, super disintegrants sodium starch glycolate and Croscarmellose sodium were used $4 \mathrm{mg}$ per tablet. The results of drug release from IR layer, infers that at the concentration of $4 \mathrm{mg}$ both the super disintegrants comply with the limit for drug release in $30 \mathrm{~min}(\mathrm{Table}: 9$, fig 4). In the formula for Valsartan sustained release layer, Hydroxypropyl Methyl Cellulose (HPMC K 100 M) and HPMC $\mathrm{K} 4 \mathrm{M}$ were used as retardant polymers. From the drug release profile it infers that Hydroxypropyl Methyl Cellulose (HPMC K $100 \mathrm{M}$ ) at a concentration of 50 t0 60\% produced desired release profile for Valsartan extended release layer as per USP limits. B3 was considered the best formulation based on the release profile of both the drugs in immediate and sustained release layer. (Table: 10 , fig $3 \& 3 \mathrm{~A}$ ). To analyze the pattern of drug release, the drug release data of the best formulations were subject to release kinetics studies. (Table 10) The results show that formulation B3 depicts zero order release pattern in which the prime mechanism is diffusion controlled release. B3 optimized tablets was selected for accelerated stability studies for a period of one month at $40^{\circ} \mathrm{C} / 75 \% \mathrm{R}$. as per in-house specifications. The stability studies confirmed that there was no significant difference over the testing period with respect to dissolution profile, assay and other physical parameters thus satisfying in-house specifications. In vivo pharmacokinetic studies in Rabbit animal model was carried out for best formulation B3.The pharmacokinetic parameters are summarized in table 11 .

\section{CONCLUSION:}

From present study, it can be concluded that bilayer tablet dosage form is an ideal fixed dosage form for formulating combination of drugs. Bilayer tablet containing Nebivolol Hydrochloride and Valsartan in immediate release and sustained release layers respectively would be a novel concept in treating hypertension. I formulation point of view precompression and Post compression parameters suggests that, this formulae of drug and excipient combination would provide a satisfactory results.

The immediate release layer of Nebivolol Hydrochloride helps in the immediate release of the drug which acts as loading dose to reduce hypertension initially. The modified release of Valsartan in Sustained release layer is helpful in maintaining the drug levels above the minimum required concentration for a long duration. This reduces dosing frequency from twice a day to once a day. Hence this technology can be used to formulate fixed dosage form of Nebivolol Hydrochloride and Valsartan which can give an 
optimal therapy for hypertension. The IVIVC suggests a moderate linearity between the In vitro and In vivo results. These findings could be substantiated by further pharmacodynamic evaluation.

\section{AUTHOR CONTRIBUTION STATEMENT}

I hereby declare that this part of the research work is an extension to the preliminary work on the same ideology reflected earlier. Hence the need for further s studies and the novelty in the present research can substantiate the current work. The corresponding author has designed and performed the experiments, analyzed the data and manuscript writing. Co-authors have reviewed the experimental work and manuscript.

\section{ACKNOWLEDGEMENTS}

The author is sincerely thankful to KMS Pharma Formulation development Healthcare Pvt Ltd, Chennai for providing facility for this research work.

\section{CONFLICT OF INTEREST}

Conflict of interest declared none.

\section{REFERENCES:}

1. Suresh P Vyas, Roop K Khar(2012) In: Pharmacokinetic basis of controlled drug delivery : concepts and advances.2nd edition.

2. Bartosh SM, Aronson AJ, Childhood hypertension an update on etiology, diagnosis and treatment. Paediatr clin North AM. 1999,46(2):235-252

3. Girerd X, Hanon O, Pannier B: Trends in the use of antihypertensive drugs in France from 2002 to 2012: FLHAS surveys. Ann Cardiol Angeiol 2013; 62:210 -2014.

4. Anonymous. 1999 WHO _ International society of Hypertension guide lines for management of Hypertension. Guidelines sub- committees. J Hypertns 1999; 17: 151 - 183.

5. Fagan TG: Remembering the lessons of basic Pharmacology. Arch Intern Med 1994; 154: 1430- 1431.
6. Pudipeddi $\mathrm{M}$; Biopharmaceutical challenges of fixed dose combination product development.AM .Pharm Rev 2007; 10: 120-126.

7. Formulation and evaluation of valsartan sr tablets using hydrophilic and hydrophobic polymer L. P. Hingmire* , D. M. Sakarkar Dept. of Pharmacy, Jagdish Prasad Jhabarmal Tibrewala University, Chudela, Dist.Jhunjhunu, Rajasthan, India *Corresponding author: E-mail: lax_hingmire@rediffmail.com, phone no.+91-9422169470

8. Robert Weiss; Vascular Health and Risk Management 2006:2(3) 303-308. (C) 2006 Dove Medical Press Limited

9. Thomas D. Giles, John R. Cockcroft, Bertram Pitt, Abhijeet Jakate, and Harold M. Wright: Rationale for Nebivolol/valsartan combination for hypertension: review of preclinical and inical data. Journal of Hypertension, Volume 35 _ Number 9_September 2017

10. Martin A. Swarbrick, J and Cammarata. A., Physical Pharmacy, 2nd Edition, 1983: P.532-533, 502, 513-515.

11. Harika Ryakala, S. Dineshmohan, Alluri Ramesh, and V. R. M. Gupta, "Formulation and In Vitro Evaluation of Bilayer Tablets of Nebivolol Hydrochloride and Nateglinide for the Treatment of Diabetes and Hypertension," Journal of Drug Delivery, vol. 2015, Article ID 827859, 14 pages, 2015 https://doi.org/10.1155/2015/827859.

12. Indian Pharmacopoeia, Govt of India.Ministry of Health and family welfare by Controller of publications, Delhi; 1996, Vol.I, Anx-144-145. Fourth edition, 3(37), P.734-736.

13. P.R. 168 Sharma, S.A. Lewis, Design and invitro/invivo evaluation of extended release matrix tablets of Natelinide Journal of Young Pharmacists 5 (2013) 167e172

14. Yang SG. Biowaiver extension potential and IVIVC for BCS Class II drugs by formulation design: case study for cyclosporine self-micro emulsifying formulation. Arch Pharm Res. 2010;33(11):1835-1842

15. Emami J. In vitro-in vivo correlation: from theory to applications. J Pharm Pharm Sci. 2006;9(2):169-189.

16. Aulton M.E, 1988.Pharmaceutics: The science of dosage form, London, Churchill livingstone,N.Y; P.124-127, 215-222, $247-$ 248.

17. Izhar Kasid et al :Development of bilayer tablets of lisinopril and gliclazide: Development of bilayer tablets of lisinopril and gliclazide: In vitro and in vivo evaluation Der Pharmacia Lettre, 2013, 5 (2):54-62 